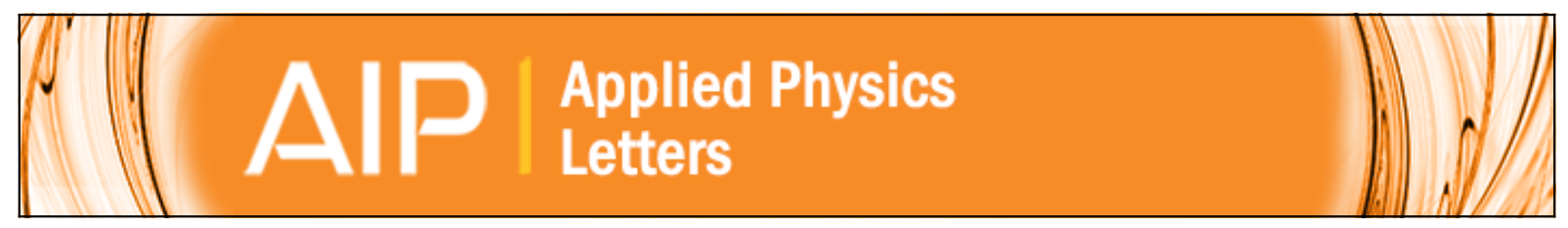

\title{
Electrical transients in the ion-beam-induced nitridation of silicon
}

Mladen Petravic and Prakash N. K. Deenapanray

Citation: Applied Physics Letters 78, 3445 (2001); doi: 10.1063/1.1376661

View online: http://dx.doi.org/10.1063/1.1376661

View Table of Contents: http://scitation.aip.org/content/aip/journal/apl/78/22?ver=pdfcov

Published by the AIP Publishing

\section{Articles you may be interested in}

Proximity effect in ion-beam-induced deposition of nanopillars

J. Vac. Sci. Technol. B 27, 1838 (2009); 10.1116/1.3155825

Ion-beam-induced amorphization and recrystallization in silicon

J. Appl. Phys. 96, 5947 (2004); 10.1063/1.1808484

Dynamics of the ion beam induced nitridation of silicon

J. Vac. Sci. Technol. A 20, 1261 (2002); 10.1116/1.1481045

Transient enhanced diffusion after laser thermal processing of ion implanted silicon

Appl. Phys. Lett. 75, 3659 (1999); 10.1063/1.125420

Kinetics of ion-beam-induced interfacial amorphization in silicon

J. Appl. Phys. 82, 5360 (1997); 10.1063/1.366458

\section{Model PS-100}

Tabletop Cryogenic Probe Station

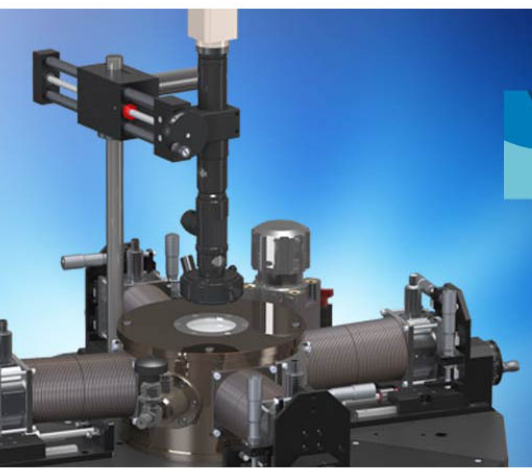

\section{Lake Shore} CRYOTRONICS

An affordable solution for a wide range of research 


\title{
Electrical transients in the ion-beam-induced nitridation of silicon
}

\author{
Mladen Petravic ${ }^{\text {a) }}$ and Prakash N. K. Deenapanray \\ Department of Electronic Materials Engineering, Research School of Physical Sciences and Engineering, \\ Australian National University, Canberra, ACT 0200, Australia
}

(Received 14 December 2000; accepted for publication 4 April 2001)

\begin{abstract}
We have studied the dynamics of the initial stages of silicon nitride formation on silicon surfaces under nitrogen beam bombardment in the secondary ion mass spectrometry apparatus. We have shown that the secondary ion signal exhibits damped oscillations below the critical impact angle for nitride formation. We have described this oscillatory response by a second-order differential equation and argued that it is initiated by some fluctuations in film thickness followed by the fluctuations in surface charging. (c) 2001 American Institute of Physics.
\end{abstract}

[DOI: $10.1063 / 1.1376661]$

Ion-induced formation of surface compounds involves several, often competing processes, determined primarily by the ion energy and angle of incidence. ${ }^{1}$ Recently, we have shown that the bombardment of $\mathrm{Si}$ with oxygen ions in a few $\mathrm{keV}$ range can produce stoichiometric oxide layers with the thickness closely related to the projected range of oxygen ions in $\mathrm{Si}^{2,3}$ The formation of nitride films under bombardment with nitrogen ions shows similar effects, as nitrogen and oxygen have similar masses and ranges in $\mathrm{Si}$, show similar sputtering effects and both have the ability to form chemical compounds with $\mathrm{Si}^{4,5}$ However, the large electrical and structural differences between oxide and nitride films contribute to the different dynamics of oxygen and nitrogen atoms accumulation under the $\mathrm{Si}$ surface and diffuison during ion bombardment.

In the present letter, we report on the dynamics of initial stages in nitride film formation under low-energy nitrogen ion bombardment. All measurements were performed in a quadrupole-type secondary ion mass spectrometry (SIMS) apparatus (Riber MIQ 256) in which the ion-beam energy and angle of incidence can be changed independently. In SIMS, the removal of surface atoms by energetic ion impact, known as sputtering, at the one time provides the analytical signal and erodes the surface to give information about the in-depth distribution of impurities. This unique feature of the SIMS technique enabled us to monitor the response of the system (i.e., the current of secondary ions sputtered from the surface) simultaneously with the build up of nitrogen atoms below the Si surface and the formation of a buried nitride layer and its extension to the surface. In that way, we were able to follow the dynamics of nitride formation from the early stages (within the transient region when surface composition changes from pure $\mathrm{Si}$ to $\mathrm{Si}$-nitride), well before the dynamic equilibrium was established. Here, the dynamic equilibrium is defined by the balance between the erosion of the nitride layer at the surface by sputtering and its growth at the internal interface by the implantation of nitrogen ions.

In Fig. 1 we show the signal of nitrogen and silicon, represented by the ${ }^{14} \mathrm{~N}^{+}$and ${ }^{28} \mathrm{Si}^{+}$ions, respectively, within the transient region for $10 \mathrm{keV} \mathrm{N}_{2}^{+}$bombardment of Si sur-

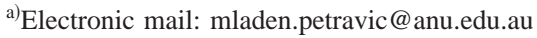

face at normal incidence. We stress, once again, that the curves in Fig. 1 reflect the dynamic evolution of the Si surface under nitrogen bombardment as both signals were collected during the formation of a buried nitride layer and its extension to the surface by sputtering. Before reaching a constant level (for $t>550 \mathrm{~s}$ ), both signals exhibit several oscillations, more intensive in the case of nitrogen signal.

Recently, we have shown that the bombardment of $\mathrm{Si}$ with nitrogen ions at near-normal incidence eventually transforms the surface into a continuous nitride layer. ${ }^{5}$ The critical angle for nitride formation (measured from the surface normal) depends on the nitrogen-beam energy. This effect is shown in more detail in Fig. 2 giving the dependence of nitrogen build-up profiles on the primary beam angle of incidence for $13.5 \mathrm{keV} \mathrm{N}_{2}^{+}$bombardment. As the impact angle increases from $0^{\circ}$ to $45^{\circ}$, oscillations become less intensive and eventually disappear above $30^{\circ}$. This behavior correlates nicely with the angular dependence of the nitrogen content determined from the Rutherford backscattering spectra taken from the bottom of the SIMS craters produced by $13.5 \mathrm{keV}$ $\mathrm{N}_{2}^{+}$bombardment at different impact angles. ${ }^{6}$ For angles less than about $30^{\circ}$, N-rich nitride layer is formed at the Si surface (with the ratio of $\mathrm{N}$ to $\mathrm{Si}$ of about 2.3), whereas at

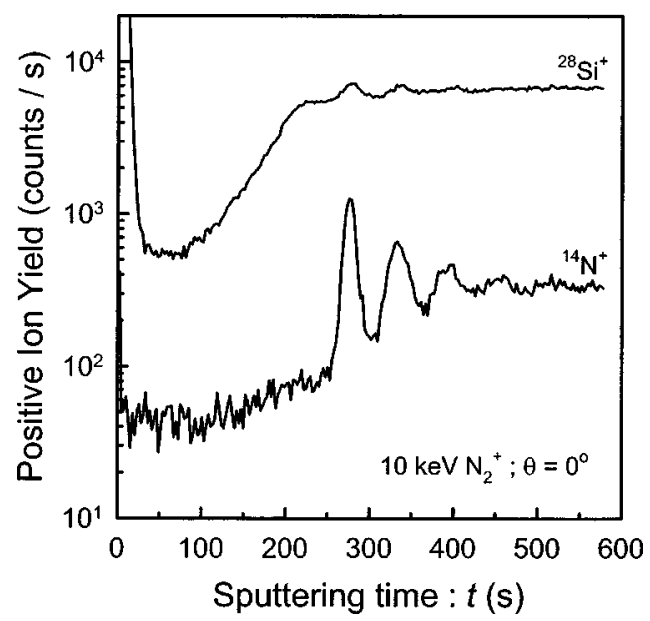

FIG. 1. Buildup profiles of nitrogen and silicon, represented by ${ }^{14} \mathrm{~N}^{+}$and ${ }^{28} \mathrm{Si}^{+}$ions, respectively, measured in the transient region during $10 \mathrm{keV} \mathrm{N}_{2}^{+}$ bombardment of $\mathrm{Si}$ at normal incidence. 


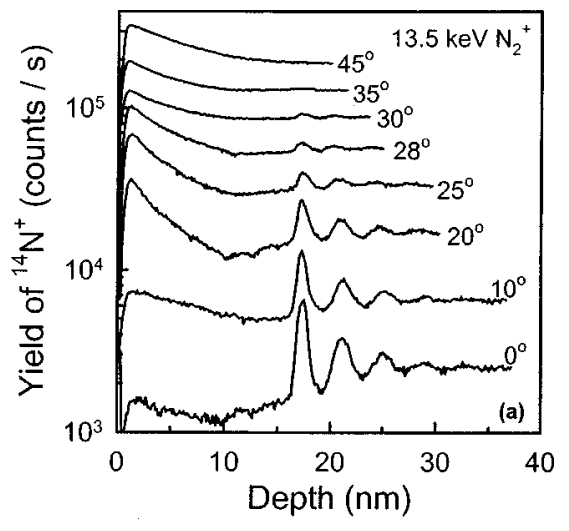

FIG. 2. Nitrogen signal measured during bombardment of Si with $13.5 \mathrm{keV}$ $\mathrm{N}_{2}^{+}$ions at different angles of incidence.

higher angles, the combination of sputtering and depth of the implanted nitrogen limits the composition. ${ }^{6}$

The initial stage of nitride formation is similar to that of oxide formation. ${ }^{2,4,5}$ Briefly, with increasing nitrogen dose, the nitrogen concentration builds up below the surface, with no apparent movement of $\mathrm{N}$ in $\mathrm{Si}$, until a buried nitride layer is formed. At this stage, the difference between the oxygen and nitrogen induced effects becomes significant. Firstly, a dramatic densification during nitride formation (density of $\mathrm{Si}_{3} \mathrm{~N}_{4}$ is $1.03 \times 10^{23}$ atoms $/ \mathrm{cm}^{3}$ ) causes a substantial reduction in ion ranges in $\mathrm{Si}$-nitride compared with $\mathrm{Si}$ : $125 \AA$ and $195 \AA$, respectively, for $13.5 \mathrm{keV} \mathrm{N}_{2}^{+}$bombardment (shown in Fig. 2) from the TRIM'95 code. ${ }^{7}$ Secondly, the low diffusivity of nitrogen in $\mathrm{Si}$-nitride contributes to the build up of nitrogen atoms within the buried nitride layer. On the other hand, there is a strong tendency for nitrogen to form $\mathrm{N}_{2}$ and hence, the high dose nitrogen implantation may result in formation of a gas-phase $\mathrm{N}_{2}$ bubbles near the projected range of nitrogen ions in $\mathrm{Si}^{8}$ Finally, it is well known that the insulating surfaces, such as oxides and nitrides, will charge positively under ion bombardment. ${ }^{9}$ However, the surface charging on nitride surfaces may be several times higher than on oxide surfaces. ${ }^{10}$ Any change of the surface charge will change the signal of secondary ions sputtered from the surface. Indeed, the surface charging plays an important role in development of oscillatory response, as illustrated in Fig. 3. Samples coated with thin Au films (10-20 nm) have shown reduced, or even completely suppressed, oscillations. Coating the surfaces of insulating samples with a thin Au layer is a standard procedure in SIMS which reduces surface charging by greatly improving the leakage path for charge from the instantaneous crater surface to the sample holder. ${ }^{11}$

Another important feature during the transient stages of nitride formation is the change in thickness of the surface nitride layer. ${ }^{5}$ We argue that this change in thickness initiates the oscillatory response. As mentioned earlier, the range of nitrogen ions in $\mathrm{Si}_{3} \mathrm{~N}_{4}$ is only $\sim 65 \%$ of the range in $\mathrm{Si}$ (and even less for the overstoichiometric layers) for the ion energies used in our experiments. Consequently, upon formation of a nitride layer continuous to the surface, the range of $\mathrm{N}$ ions is drastically reduced below the thickness of the nitride layer, contributing to the accumulation of nitrogen within the nitride film (due to the low diffusivity of $\mathrm{N}$ in nitrides) and possibly the formation of $\mathrm{N}_{2}$ bubbles. Further ion bombard-

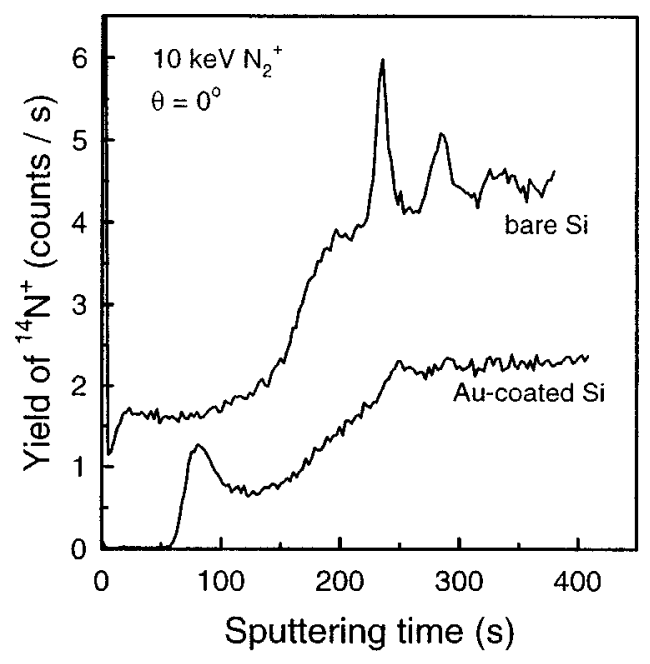

FIG. 3. Nitrogen signal measured from a clean and an Au-coated Si surface during $10 \mathrm{keV} \mathrm{N} \mathrm{N}_{2}^{+}$bombardment at normal incidence.

ment and sputtering of the surface reduces the film thickness to the one comparable to the range of $\mathrm{N}$ in $\mathrm{Si}$-nitride (or, more precisely, to the range plus straggling). At this stage, $\mathrm{N}$ ions start to penetrate again into the underlying $\mathrm{Si}$, but much deeper than they would into the $\mathrm{Si}$ nitride, giving rise to the increase of film thickness slightly above the range of $\mathrm{N}$ atoms in $\mathrm{Si}$-nitride. Now again, the film thickness is above the range of $\mathrm{N}$ ions in $\mathrm{Si}$-nitride and the whole process repeats itself. In other words, the combination of sputtering and the change in penetration depth of $\mathrm{N}$ atoms from $\mathrm{Si}$ to $\mathrm{Si}$-nitride opposes the change of film thickness and tries to keep it constant. When the film becomes too thick, sputtering reduces its width. When the film becomes too thin, $\mathrm{N}$ atoms penetrate into underlying $\mathrm{Si}$, react with $\mathrm{Si}$ and increase the film thickness. This situation is analogous to the action of an inductor in an electrical circuit. If the current through an inductor changes, the inductor physical voltage opposes the change tending to keep the current constant. However, the variation in film thickness eventually dies out and a steady state situation prevails. The "inductance" of nitride layers becomes less pronounced at higher bombardment angles (see Fig. 2) or lower impact energies (not shown), ${ }^{6}$ as the higher sputtering rates (number of sputtered atoms per incident ion, which increases with the impact angle) or the lower initial film thickness (which decreases with lowering the impact energy) limit fluctuations of the film thickness.

Any change in thickness of the nitride layer will cause the change in surface charging and, consequently, the change of secondary ion signal (the thinner the layer, the less positive charging on the surface, and the higher the signal of secondary ions). Therefore, we have used the second-order differential equation of an electrical oscillator ${ }^{12}$ to describe the oscillations in $\mathrm{N}^{+}$signal caused by the oscillations of the surface charge:

$$
\ddot{q}+2 \zeta \omega_{0} \dot{q}+\omega_{0}^{2} q=F_{0} .
$$

Here, $\zeta$ is the damping factor, $\zeta=(R / 2) \sqrt{C / L}, \omega_{0}$ is the natural frequency of the circuit, $\omega_{0}=1 / \sqrt{L C}$, and $q$ represents the charge on the capacitor. $F_{0}$ is the constant initial impulse (analogous to the constant primary beam current). 


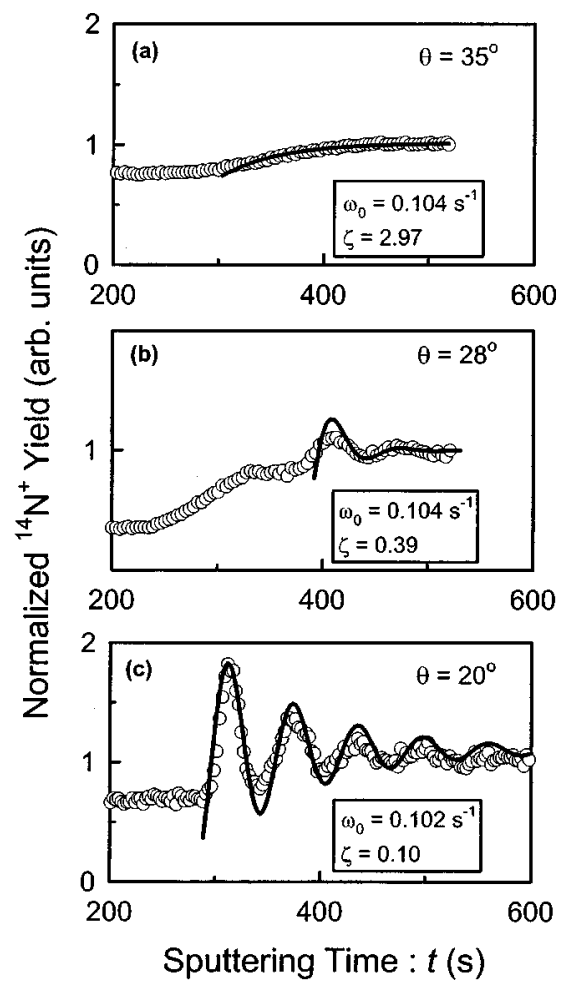

FIG. 4. Buildup profiles of nitrogen (open circles) obtained at different angles of incidence during $10 \mathrm{keV} \mathrm{N} \mathrm{N}_{2}^{+}$bombardment of Si. Solid lines are fits of experimental curves to (a) Eq. (2) and (b), (c) Eq. (3).

For $\zeta>1$, the system is overdamped and the solution of Eq. (1) has the form: ${ }^{12}$

$$
\begin{aligned}
\frac{q}{F_{0}}= & 1-\exp ^{-\zeta \omega_{0} t}\left[\cosh \left(\omega_{0} t \sqrt{\zeta^{2}-1}\right)\right. \\
& \left.+\frac{\zeta}{\sqrt{\zeta^{2}-1}} \sinh \left(\omega_{0} t \sqrt{\zeta^{2}-1}\right)\right] .
\end{aligned}
$$

For $\zeta<1$, the system is underdamped with the solution: ${ }^{12}$

$$
\begin{aligned}
\frac{q}{F_{0}}= & 1-\exp ^{-\zeta \omega_{0} t}\left[\cos \left(\omega_{0} t \sqrt{1-\zeta^{2}}\right)\right. \\
& \left.+\frac{\zeta}{\sqrt{1-\zeta^{2}}} \sin \left(\omega_{0} t \sqrt{1-\zeta^{2}}\right)\right] .
\end{aligned}
$$

As an example, we show in Fig. 4 the experimental results for $10 \mathrm{keV} \mathrm{N} \mathrm{N}_{2}^{+}$bombardment (open circles), together with the fitting (full line) to Eqs. (2) and (3). The fitting parameters are also shown in Fig. 4. As the position of the first oscillatory peak in Fig. 2 is close to the projected range of $\mathrm{N}$ ions in $\mathrm{Si}$, we have adjusted the initial time for simulations in Fig. 4 to correspond to the moment when the buried nitride layer intersects the surface.
The absence of any oscillatory responses at $35^{\circ}$ (which is above the critical angle, $\theta_{\mathrm{cr}}$, for the formation of an overstoichiometric nitride layer) indicates the low quality factor, $Q \sim 1 / \zeta \sim \sqrt{L / R^{2} C}$, of the oscillatory circuit. The oscillations start to appear at $\theta_{\text {cr }}$ and we can fit them by the Eq. (3), as shown in Figs. 4(b) and 4(c) for bombardment at $28^{\circ}$ and $20^{\circ}$, respectively. Indeed, Eq. (3) describes our experimental data rather well. The change of the intensity and damping of oscillations below $\theta_{\text {cr }}$ [Figs. 2, 4(b), and 4(c)] may be rationalized in the following way. For these low angles, the thickness of the nitride layer does not change significantly with the angle, while the stoichiometry remains almost the same. ${ }^{6}$ Therefore, all changes observed in the oscillation behavior for these low angles simply reflect some variations of the "inductive" character of nitride layers. We argue that the lower sputtering rates and initially thicker nitride films at angles closer to the normal ${ }^{3}$ effectively extend the equilibration time, giving more "inductance" (or, equivalently, the higher $Q$ ) to nitride layers.

In conclusion, we have studied the initial stages of ionbeam-induced formation of silicon-nitride and observed some damped oscillations in the secondary ion signal from thin surface nitride layers. We have shown that these oscillations can be modeled by the second-order differential equation of a dissipative electrical circuit under constant excitation.

The authors acknowledge helpful discussions with Henri Budinov.

\footnotetext{
${ }^{1}$ See, for example, N. Herbots, O. C. Hellman, P. Ye, X. Wang, and O. Vancauwenberghe, in Low Energy Ion-Surface Interactions, edited by J. W. Rabalais (Wiley, New York, 1994), p. 389.

${ }^{2}$ J. S. Williams, M. Petravic, B. G. Svensson, and M. Conway, J. Appl. Phys. 76, 1840 (1994).

${ }^{3}$ P. N. K. Deenapanray and M. Petravic, Surf. Interface Anal. 27, 92 (1999).

${ }^{4}$ W. De Coster, B. Brijs, H. Bender, J. Alay, and W. Vandervorst, Vacuum 45, 389 (1994).

${ }^{5}$ M. Petravic, J. S. Williams, M. Conway, and P. N. K. Deenapanray, Appl. Phys. Lett. 73, 1287 (1998).

${ }^{6}$ P. N. K. Deenapanray and M. Petravic (unpublished).

${ }^{7}$ J. F. Zigler, J. P. Biersack, and U. Littmark, The Stopping and Range of Ions in Solids (Pergamon, New York, 1986), Vol. 1.

${ }^{8}$ C. D. Meekison, G. R. Booker, K. J. Reeson, P. L. F. Hemmenet, R. F. Peart, R. J. Chater, J. A. Kilner, and J. R. Davis, J. Appl. Phys. 69, 3503 (1991).

${ }^{9}$ D. V. McCaughan, R. A. Kushner, and V. T. Murphy, Phys. Rev. Lett. 30, 614 (1973).

${ }^{10}$ C. J. Vriezema and P. C. Zalm, Surf. Interface Anal. 17, 875 (1991).

${ }^{11}$ D. S. McPhail, M. G. Dowsett, and E. H. C. Parker, J. Appl. Phys. 60, 2573 (1986)

${ }^{12}$ See, for example, P. O. A. L. Davies, An Introduction to Dynamic Analysis and Automatic Control (Wiley, New York, 1965), p. 111.
} 